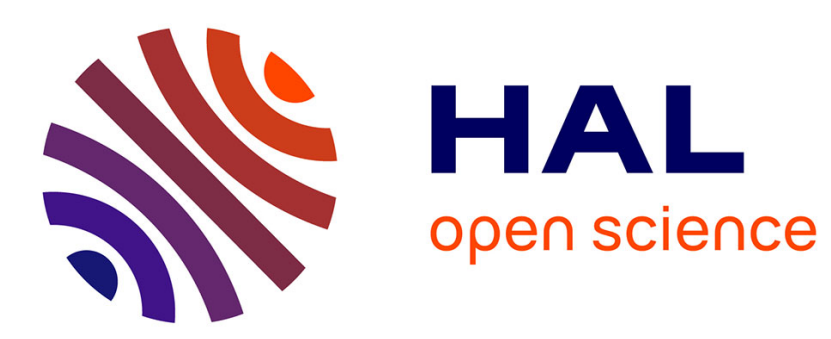

\title{
Continual Learning for Dense Labeling of Satellite Images
}

\author{
Onur Tasar, Yuliya Tarabalka, Pierre Alliez
}

\section{To cite this version:}

Onur Tasar, Yuliya Tarabalka, Pierre Alliez. Continual Learning for Dense Labeling of Satellite Images. IGARSS 2019 - IEEE International Geoscience and Remote Sensing Symposium, Jul 2019, Yokohama, Japan. hal-02276543

\section{HAL Id: hal-02276543 \\ https://hal.inria.fr/hal-02276543}

Submitted on 2 Sep 2019

HAL is a multi-disciplinary open access archive for the deposit and dissemination of scientific research documents, whether they are published or not. The documents may come from teaching and research institutions in France or abroad, or from public or private research centers.
L'archive ouverte pluridisciplinaire HAL, est destinée au dépôt et à la diffusion de documents scientifiques de niveau recherche, publiés ou non, émanant des établissements d'enseignement et de recherche français ou étrangers, des laboratoires publics ou privés. 


\title{
CONTINUAL LEARNING FOR DENSE LABELING OF SATELLITE IMAGES
}

\author{
Onur Tasar $^{1}$, Yuliya Tarabalka ${ }^{1,2}$, Pierre Alliez $^{1}$ \\ ${ }^{1}$ Université Côte d'Azur, Inria, TITANE team, France; ${ }^{2}$ LuxCarta Technology, France \\ Email: \{firstname.lastname\} @inria.fr
}

\begin{abstract}
In dense labeling problem, the major drawback of the convolutional neural networks is their inability to learn new classes without affecting performance for the old classes on the data, having no annotations for the previous classes. In this work, we address the issue of adding new classes continually to the already trained network from a stream of data. Our approach comprises two main components: adaptation and remembering. For adaptation, we keep a clone of the previously trained network, which serves as a memory for the old classes in absence of their annotations on the new data. The updated network learns new as well as old classes on the current data using output of the memory network and the new groundtruth. For remembering, we store a little portion of the previous data, from which we systematically feed samples to the updated network during training. Our results prove that segmentation capabilities for the new classes can be added to the already trained network without catastrophically forgetting the previously learned information.
\end{abstract}

Index Terms - Continual learning, dense labeling, semantic segmentation, convolutional neural networks, catastrophic forgetting

\section{INTRODUCTION}

With the great improvements of satellite sensors, it has been possible to collect massive amount of data, which has opened the door to a wide range of remote sensing applications such as navigation, disaster prevention, and mapping. Over the years, because of the growing interest in such applications, generating maps from satellite images automatically has been one of the most popular topics in remote sensing community. Although it has been shown that convolutional neural networks (CNNs), particularly U-net and its variants [1], can generate high quality maps, most of the works in the literature are limited to learning from only static training data. Since new images are collected from all around the world everyday, one can not assume that all the training images available in the beginning of the training procedure. In addition, annotations obtained from different sources usually have distinct

The authors would like to thank CNES and ACRI-ST for initializing and funding this study. The authors also thank Luxcarta (https://luxcarta.com) for providing the annotated data. classes. Moreover, it may not always possible to store huge amount of data. Because of these reasons, it is of paramount importance to devise a classification system that can learn new classes continually while retaining performance for the old classes without accessing the entire previous data. The continual learning problem is illustrated in Fig. 1. To the best of our knowledge, in remote sensing community, this problem has not been explored yet.

The main drawback of the common CNNs for the continual learning problem is that their performance for the old classes significantly drops when new classes are added to the trained model on the data, having no ground-truth for the previous classes. The aforementioned abrupt performance loss is referred as catastrophic forgetting in the literature [2]. To overcome this problem, there have been some attempts, which change the network structure. An example methodology that is in this category is described in [3], where the network is expanded as new classes are added. The obvious limitation of this approach is that the network grows every time new classes are taught. Since deep neural networks contain millions of parameters, different configurations of the parameters may produce the same results. Inspired from this idea, several works, which try to determine the important neurons and prevent them from changing drastically, have been proposed $[4,5]$. The methodology proposed in [6] uses a small fraction of the previous training data to prevent forgetting the already learned information. The knowledge distillation from one network to another one [7] gave inspiration to several works. In $[8,9]$, the previous knowledge is distilled from the formerly trained network to the current one on the new data. Even though several methodologies have been proposed to overcome catastrophic forgetting, none of those works investigates continual learning for dense labeling problem.

In this paper, we propose a method, which enables to learn dense labeling capabilities for the new classes without forgetting the old classes even if the whole previous training data are not available.

\section{METHODOLOGY}

Our network structure is U-net, comprising an encoder that is architecturally the same as encoder of VGG-16 [10] and a symmetric decoder. Each convolution and deconvolution operation is followed by a rectified liner unit (ReLU). Since 

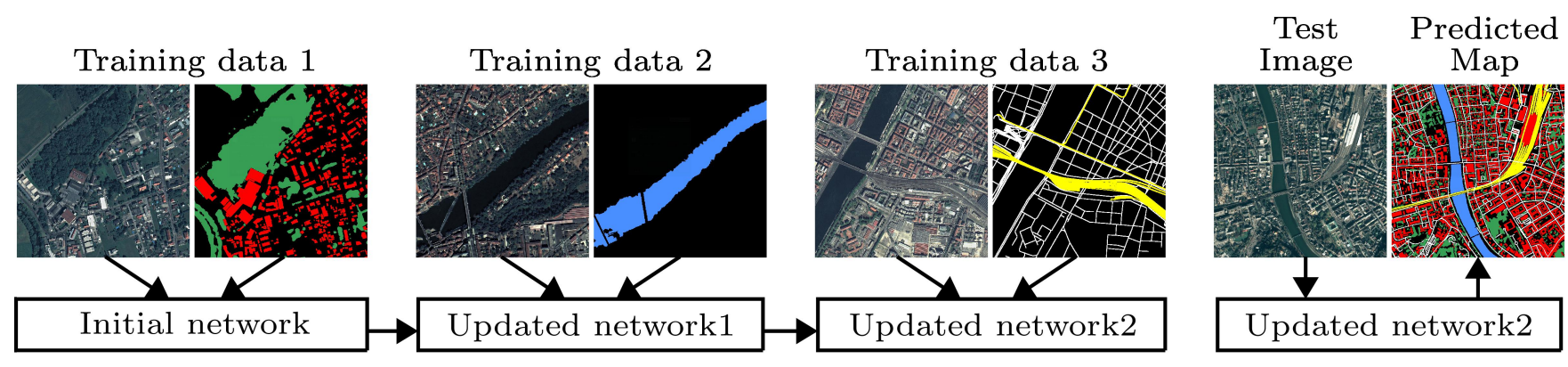

Fig. 1. Continual learning problem. Every time new training data are retrieved, only a small potion of the previous ones are stored. The classes are building (red), tree (green), water (blue), road (white), and railway (yellow).

batch normalization uses the memory inefficiently, we prefer not to use it. The output of our network consists of binary predicted maps for all the classes.

Let us assume that the current training data are denoted by $D_{\text {curr }}$. The sets of previously learned classes and the classes in $D_{\text {curr }}$ are indicated by $\mathcal{L}_{\text {prev }}$ and $\mathcal{L}_{\text {curr }}$, where $\mathcal{L}_{\text {prev }} \cap \mathcal{L}_{\text {curr }}=\emptyset$. During adaptation, we teach $\mathcal{L}_{\text {curr }}$ and $\mathcal{L}_{\text {prev }}$ to the network on $D_{\text {curr }}$. Since the annotations for $\mathcal{L}_{\text {prev }}$ are not available in $D_{\text {curr }}$, we transfer the knowledge from the memory network to the updated network. We denote by $\mathbf{y}_{\text {curr }}$, the binary target vectors of the pixels in a training batch from $D_{\text {curr }}$ for all the classes. We denote the binary predicted probabilities for $\mathcal{L}_{\text {curr }}$ and $\mathcal{L}_{\text {prev }}$ from the updated network by $\hat{\mathbf{y}}_{\text {up_curr }}$ and $\hat{\mathbf{y}}_{\text {up_prev }}$, respectively. The predictions for $\mathcal{L}_{\text {prev }}$ from the memory network are indicated by $\hat{\mathbf{y}}_{\text {mem }}$. To learn $\mathcal{L}_{\text {curr }}$, we minimize the classification loss $L_{\text {sigm }}^{\text {class }}\left(\hat{\mathbf{y}}_{\text {up_curr }}, \mathbf{y}_{\text {curr }}\right)$, which is the sigmoid cross entropy loss between $\hat{\mathbf{y}}_{\text {up_curr }}$ and $\mathbf{y}_{\text {curr }}$. In order to distill the knowledge for $\mathcal{L}_{\text {prev }}$ from the memory network to the updated network, we minimize the distillation loss

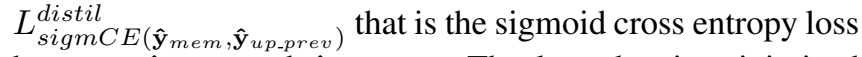
between $\hat{\mathbf{y}}_{\text {mem }}$ and $\hat{\mathbf{y}}_{\text {up_prev }}$. The loss that is minimized during adaptation is defined as:

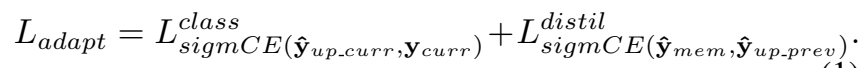

For remembering, we store a little fraction of the previous training data. Because in satellite images number of samples for the classes are usually imbalanced, randomly selecting the training patches that would be stored might result in having no samples for the less frequent classes. Hence, we handle the class imbalance problem. Let us assume that $D_{\text {prev }}^{(j)}$ is the $j^{\text {th }}$ previous training data, and $\mathcal{L}_{\text {prev }}^{(j)}$ denotes the classes in $D_{\text {prev }}^{(j)}$. We compute a weight $w_{c}$ for each class $c \in \mathcal{L}_{\text {prev }}^{(j)}$ in $D_{\text {prev }}^{(j)}$ as:

$$
w_{c}=\frac{\operatorname{median}\left(f_{c} \mid c \in \mathcal{L}_{\text {prev }}^{(j)}\right)}{f_{c}},
$$

where $f_{c}$ is frequency of the pixels, belonging to class $c$. We then compute an importance value $I^{(l)}$ for the $l^{t h}$ training

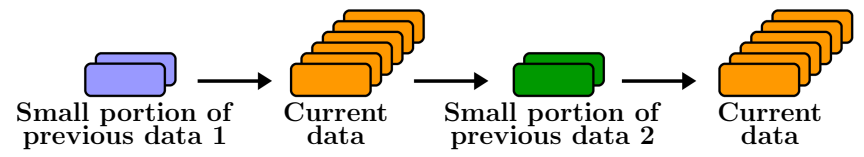

Fig. 2. Example optimization sequence. The sequence is: $L_{\text {rem }}$ on data $1, L_{\text {adapt }}$ on the current data, $L_{\text {rem }}$ on data 2 , and $L_{\text {adapt }}$ on the current data again.

patch in $D_{\text {prev }}^{(j)}$ as:

$$
I^{(l)}=\sum_{c \in \mathcal{L}_{p r e v}^{(j)}} w_{c} f_{c}^{(l)},
$$

where $f_{c}^{(l)}$ denotes the number of pixels that are labeled as class $c$ in the patch. We store a certain number of patches, having the highest $I$ values. In order to prevent storing only the patches that are located in very close geographic locations, we also store certain number of randomly selected patches. The number of patches that are selected randomly and using $I$ values have to be determined. Let us assume that $\mathbf{y}_{\text {prev }}^{(j)}$ is the target vector, and $\hat{\mathbf{y}}_{\text {up_prev }}^{(j)}$ denotes the predictions of the pixels for $\mathcal{L}_{\text {prev }}^{(j)}$ in the training batch from the stored, small portion of $D_{\text {prev }}^{(j)}$. The remembering loss $L_{r e m}$ is defined as:

$$
L_{\text {rem }}=L_{\text {sigmCE }}\left(\mathbf{y}_{\text {prev }}^{(j)}, \hat{\mathbf{y}}_{\text {up_prev }}^{(j)}\right) \text {, }
$$

where $L_{\text {sigmCE }}\left(\mathbf{y}_{\text {prev }}^{(j)}, \hat{\mathbf{y}}_{\text {up_prev }}^{(j)}\right)$ is the sigmoid cross entropy

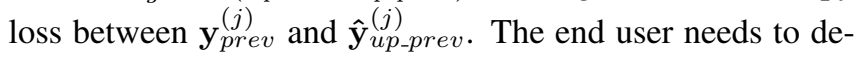
termine how frequently and on which previous training data, the samples would be fed to the network to optimize $L_{r e m}$. An example optimization sequence is depicted in Fig. 2.

\section{METHODS USED FOR COMPARISON}

We compare our methodology with the following approaches:

Multiple learning : In this approach, we train a new network from scratch every time new training data are retrieved. Because of the growing number of classifiers, this learning approach is inefficient in terms of storage. Besides, since the 
test images have to be segmented by each trained model to generate label maps for all the classes, the running time in the test stage might be very long.

Fixed representation: Whenever new training data are obtained, we increase the number of filters in the last convolutional layer that is responsible for generating a binary predicted map for each class. In the training stage, we optimize only the filters for the new classes, and freeze the rest of the network. As we do not update the filters for the previous classes, the exact performance for them is retained. However, the network is not able to learn the new classes well, as the extracted features are not representative.

Fine-tuning : This approach is similar to fixed representation. The difference is that we freeze only the filters for the previous classes in the last convolutional layer, and optimize the rest of the network. This methodology suffers from catastrophic forgetting.

Continual learning w/o rem.: This is our approach without optimizing $L_{\text {rem }}$. Since the whole network is adapted to the last training data, the information learned from the previous data might be forgotten.

\section{EXPERIMENTS}

In our experiments, we use the Luxcarta dataset, which contains satellite images collected from 11 cities in France and 11 cities in Austria. The spatial resolution is $1 \mathrm{~m}$. The full annotations for building, tree, road, railway, and water classes are available. We use 9 cities from each country as the training data, and the rest of the cities as test. We split the training cities into three groups, as reported in Table 1 . We assume that the cities in each group have annotations for only the classes that are indicated in the table. When dividing the training cities into groups, we make sure that cities in each group have acceptable number of samples for the indicated classes. We suppose that the training data are streamed in this order: Train1, Train2, and Train3. For our approach, we store $30 \%$ of the previous training patches, in which $15 \%$ are selected randomly and the other $15 \%$ are chosen using the $I$ values. For the approaches described in Sec. 3, we do not use the previous training data.

In the pre-processing stage, we split all the training images into $384 \times 384$ patches, having $32 \times 32$ pixels of overlap between the neighboring patches. We divide the test images into $2240 \times 2240$ patches with an overlap of $64 \times 64$ pixels. After classifying each test patch, we merge them back to get the final predicted maps. For the normalization, we subtract 127 from the pixels of each spectral band.

We train 3 models for multiple learning (one from each group) for 500 epochs, where each epoch consists of 100 iterations. For the rest of the approaches, whenever new training data are retrieved, we update the previous model by training for the same number of epochs and iterations as for multiple learning. For continual learning, when we add road and railway classes on Train2 to the model trained on Train1, we op-
Table 1. Training and test cities.

\begin{tabular}{|c|c|c|}
\hline Data Type & Class & City (Country) \\
\hline Train1 & $\begin{array}{l}\text { building } \\
\text { tree }\end{array}$ & $\begin{array}{c}\text { Bad Ischl (Austria) } \\
\text { Osttirol (Austria) } \\
\text { Voitsberg (Austria) } \\
\text { Bayonne-Biarritz (France) } \\
\text { Bourges (France) } \\
\text { Draguignan (France) } \\
\text { Nîmes (France) }\end{array}$ \\
\hline Train2 & $\begin{array}{l}\text { road } \\
\text { railway }\end{array}$ & $\begin{array}{c}\text { Enns (Austria) } \\
\text { Innsbruck (Austria) } \\
\text { Klagenfurt (Austria) } \\
\text { Sankt Pölten (Austria) } \\
\text { Béziers (France) } \\
\text { Lyon (France) }\end{array}$ \\
\hline Train3 & water & $\begin{array}{c}\text { Albi (France) } \\
\text { Villach (Austria) } \\
\text { Salzburg (Austria) } \\
\text { Angers (France) } \\
\text { Douai (France) }\end{array}$ \\
\hline Test & $\begin{array}{l}\text { building } \\
\text { tree } \\
\text { road } \\
\text { railway } \\
\text { water }\end{array}$ & $\begin{array}{c}\text { Amstetten (Austria) } \\
\text { Leibnitz (Austria) } \\
\text { Lille (France) } \\
\text { Roanne (France) }\end{array}$ \\
\hline
\end{tabular}

timize $L_{\text {adapt }}$ for 4 iterations and $L_{\text {rem }}$ for 1 iteration. To add water class on Train 3 , the optimization sequence is: $L_{\text {adapt }}$ for 2 iterations, $L_{r e m}$ on Train 1 for 1 iteration, $L_{\text {adapt }}$ for 2 iterations, and $L_{\text {rem }}$ on Train 2 for 1 iteration. We use Adam optimizer to optimize parameters of the networks. The learning rate is 0.0001 and exponential decay rate for the first and the second moment estimates are 0.9 and 0.999. Each training batch consists of 12 patches. When sampling a patch, we first randomly select a country. We then choose a random city from the selected country. We finally randomly pick a training patch from the chosen city. In the training stage, we augment the training patches with 0/90/180/270 degrees of random rotation, random horizontal and vertical flips, random contrast change, and gamma correction.

F1-scores [11] of each method for all the classes are reported in Table 2. Close-ups from the generated maps for multiple learning, continual learning w/o $L_{r e m}$, and continual learning are shown in Fig. 3. Although the output of our network architecture is a binary classification map for each class, to visualize the outputs more compactly, we provide multi-class maps in the figure by assigning each pixel to the class, having the highest probability. As expected, fixed representation can not learn new classes very well, and fine-tuning catastrophically forgets the previously learned classes. For our approach, if we do not remind information from the previous data, performance for the already learned classes might 
Table 2. F1 scores on the Luxcarta dataset.

\begin{tabular}{|c|c|c|c|c|c|c|c|}
\hline Method & Epoch & Building & Tree & Road & Railway & Water & Overall \\
\hline multiple learning & 500 & 71.25 & 68.88 & 59.28 & 55.65 & 79.83 & 66.98 \\
\hline \multirow{2}{*}{ fixed representation } & 1000 & 71.25 & 68.88 & 2.71 & 0.00 & - & \\
\hline & 1500 & 71.25 & 68.88 & 2.71 & 0.00 & 0.11 & 28.59 \\
\hline \multirow{2}{*}{ fine-tuning } & 1000 & 28.91 & 0.17 & 59.30 & 60.06 & - & \\
\hline & 1500 & 27.90 & 7.71 & 0.14 & 0.01 & 90.20 & 25.19 \\
\hline \multirow{2}{*}{$\begin{array}{c}\text { continual learning } \\
\text { w/o } L_{\text {rem }}\end{array}$} & 1000 & 74.19 & 66.32 & 56.57 & 50.87 & - & \\
\hline & 1500 & 74.91 & 66.87 & 58.14 & 51.70 & 82.32 & 66.79 \\
\hline \multirow{2}{*}{ continual learning } & 1000 & 75.98 & 72.38 & 57.29 & 50.18 & - & \\
\hline & 1500 & 76.78 & 72.06 & $\mathbf{5 9 . 5 8}$ & 53.07 & 78.94 & 68.09 \\
\hline & & \multicolumn{2}{|c|}{ Training Set 1} & \multicolumn{2}{|c|}{ Training Set 2} & Training Set 3 & \\
\hline
\end{tabular}

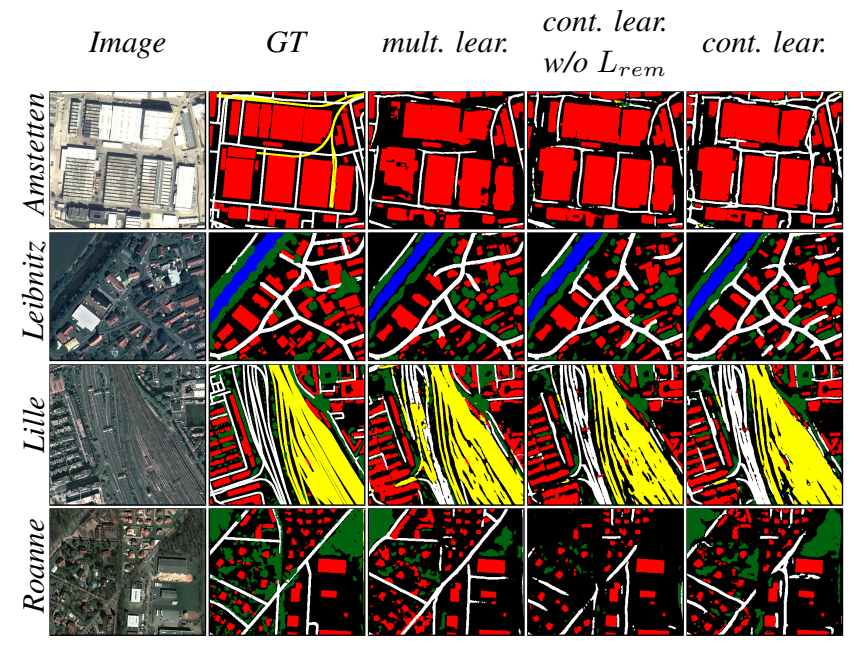

Fig. 3. Close-ups from the test cities. Classes: background (black), building (red), road (white), railway (yellow), high vegetation (green), and water (blue).

decrease over time. For instance, for tree class, performance of continual learning w/o $L_{r e m}$ is lower than multiple learning and continual learning. For multiple learning, learning for each class is limited to the images from only one group, which may cause showing a lower performance. E.g., building class is learned from only the images in Train 1 . Therefore, performance for this class is lower than our approach. As the proposed continual learning approach enables to both learn from the new data and remember from the previous data, it exhibits the best performance for most of the classes.

\section{CONCLUDING REMARKS}

We proposed a novel continual learning approach, enabling to learn segmenting new classes without deteriorating performance for the old classes, although a small portion of the previous training data are accessible. As the future work, we plan to incorporate domain adaptation techniques into the proposed continual learning approach so that new classes could be learned successfully, even when there is a large domain shift between the previous and the current training images.

\section{REFERENCES}

[1] B. Huang, K. Lu, N. Audebert, A. Khalel, Y. Tarabalka, J. Malof, A. Boulch, B. Le Saux, L. Collins, K. Bradbury, et al., "Large-scale semantic classification: outcome of the first year of inria aerial image labeling benchmark," in IEEE IGARSS, 2018.

[2] I. J. Goodfellow, M. Mirza, D. Xiao, A. Courville, and Y. Bengio, "An empirical investigation of catastrophic forgetting in gradient-based neural networks," arXiv, 2013.

[3] S. S. Sarwar, A. Ankit, and K. Roy, "Incremental learning in deep convolutional neural networks using partial network sharing," arXiv, 2017.

[4] J. Kirkpatrick, R. Pascanu, N. Rabinowitz, H. Veness, G. Desjardins, A. A. Rusu, K. Milan, J. Quan, T. Ramalho, A. Grabska-Barwinska, et al., "Overcoming catastrophic forgetting in neural networks," Proceedings of the national academy of sciences, p. 201611835, 2017.

[5] R. Aljundi, F. Babiloni, M. Elhoseiny, M. Rohrbach, and T. Tuytelaars, "Memory aware synapses: Learning what (not) to forget," arXiv, 2018.

[6] S.-A. Rebuffi, A. Kolesnikov, G. Sperl, and C. H. Lampert, "iCaRL: Incremental classifier and representation learning," in $C V P R, 2017$.

[7] G. Hinton, O. Vinyals, and J. Dean, "Distilling the knowledge in a neural network," arXiv, 2015.

[8] Z. Li and D. Hoiem, "Learning without forgetting," IEEE TPAMI, 2017.

[9] K. Shmelkov, C. Schmid, and K. Alahari, "Incremental learning of object detectors without catastrophic forgetting," in ICCV, 2017, pp. 3420-3429.

[10] K. Simonyan and A. Zisserman, "Very deep convolutional networks for large-scale image recognition," arXiv, 2014.

[11] N. Audebert, B. Le Saux, and S. Lefèvre, "Semantic segmentation of earth observation data using multimodal and multi-scale deep networks," in ACCV. Springer, 2016, pp. 180-196. 